\title{
On the knee of Galactic cosmic rays in light of sub-TeV spectral hardenings
}

\author{
Yi-Qing Guo ${ }^{1,2}$ and Qiang Yuan ${ }^{1,3}$ \\ ${ }^{1}$ Key Laboratory of Dark Matter and Space Astronomy, Purple Mountain Observatory, Chinese Academy of Sciences, Nanjing 210008, \\ Jiangsu, China; yuanq@pmo.ac.cn \\ ${ }^{2}$ Key Laboratory of Particle Astrophysics, Institute of High Energy Physics, Chinese Academy of Sciences, Beijing 100049, China; \\ ${ }^{3}$ School of Astronomy and Space Science, University of Science and Technology of China, Hefei 230026, Anhui, China
}

\begin{abstract}
More than fifty years after the discovery of the knee in the cosmic ray (CR) spectra, its physical origin remains a mystery. This is partly due to the ambiguity of the energy spectrum of individual composition. Recently, direct measurements from several space experiments found significant spectral hardenings of CR nuclei at $\sim 200 \mathrm{GV}$. A joint modeling of the direct and indirect measurements may help to understand the experimental systematics and probably the physics of the knee. In this work, we update the phenomenological "poly-gonato" model to include the spectral hardenings, with a changing spectral index of $\gamma+\beta \cdot \log E$. This modification gives a reasonable description to the CR spectra in a wide energy range. However, the fits to different data sets result in somehow different results. We find that the fit to the AMS-02 and CREAM data slightly favors a relatively low energy knee of the light components. In such a case, the expected all-particle spectra under-shoot the data, which may require an extra component of CRs. The fits to AMS-02 data and the light component $(\mathrm{H}+\mathrm{He})$ data from Tibet AS $\gamma /$ ARGO-YBJ/WFCTA or KASCADE experiments give consistent results with the all-particle spectra. We further propose a possible physical realization of such a "modified poly-gonato" model of spectral hardenings by means of spatially-dependent diffusion of CRs. We find reasonably good agreement between the model predictions and the data about CR spectra, the secondary-to-primary ratios, and the amplitude of anisotropies.
\end{abstract}

\section{Introduction}

Nearly sixty years after the discovery of the knee in the CR spectra [1], its underlying physical mechanism is still under debate [2]. It is generally accepted that each composition has its own knee and the superposition of all compositions gives the observed break of the all-particle spectra at $\sim 4 \mathrm{PeV}$. This is the so-called "poly-gonato" model [3]. The energy of the knee of each composition may be proportional to charge ( $Z$-dependent) or atomic number ( $A$-dependent), which can be used to probe the physical mechanism of the knee 2]. For example, the acceleration limit or propagation leakage may predict a $Z$-dependence of the knee of each composition [4 8]. On the other hand, an $A$-dependence may imply an interaction origin of the knee, such as the photo-disintergration [9, 10], and inelastic collisions between CRs and background photons or neutrinos [11 14].

The energy spectrum of individual nuclei composition is crucial to understand the knee puzzle. Many efforts have been paid to measure the individual spectrum with air shower experiments, however, no consensus has been achieved yet, primarily due to the systematical uncertainties of the absolute energy calibration. Some progresses in the spectral measurements of individual composition in $\mathrm{PeV}$ energies have been made in recent years with ground-based experiments. Although these measurements themselves are not completely consistent with each other, they may commonly suggest a knee below $\mathrm{PeV}$ for the light components 15 21]. Compared with the $\sim 4 \mathrm{PeV}$ knee of the all-particle spectra, such a result indicates that the knee is dominated by nuclei heavier than Helium 22 24].

The direct measurements of lower energy CRs by balloon-borne or space detectors can determine the individual spectrum much better, which were then extrapolated to high energies to bridge the direct and air shower experiments [3, 22, 23]. The extrapolation was usually based on power-law fits to the low energy data. However, remarkable spectral hardenings at an rigidity of a few hundred GV on the spectra of all major nuclei components were reported by the balloon-borne experiments ATIC-2 [25] and CREAM [26], and were confirmed with higher precision by the space detectors PAMELA [27] and AMS-02 28, 29]. Many kinds of models have been proposed to understand the origin of the spectral hardenings, including the super-position of different sources $30-32$, the non-linear acceleration of the supernova remnant shocks 33, 34], the re-acceleration mechanism when particles propagating in the Galaxy [35], and the spatially-dependent diffusion of CRs 36 -42].

Given these new measurements of both the direct and indirect experiments, we re-visit the "poly-gonato" model of CRs in this work. We tend to build an updated phenomenological model of the energy spectrum of each composition which matches these newest data. We 
adopt a log-parabolic spectrum with an asymptotically hardening spectral index of $\gamma+\beta \cdot \log E$ to describe the spectral hardenings. An exponential cutoff is employed to describe the knee of CRs. Through fitting to different data sets with the two key parameters, $\beta$ and the cutoff energy $E_{c}$, we further test the consistency among different measurements. One possible physical scenario for such a phenomenological "modified poly-gonato" model is the spatially-dependent diffusion of CRs 36]. In such a model the propagation volume was divided into two regions, the inner halo and the outer halo. The key point is that the diffusion is slower and has a shallower rigiditydependence in the inner halo than in the outer one, which can result in a break of the spectrum. As an illustration of a physical implementation of this "modified poly-gonato" model, we will also discuss this spatiallydependent diffusion scenario and compare its predictions with the observational data.

\section{2 "Modified poly-gonato" model}

\subsection{Model description}

The "poly-gonato" model to describe the knee is basically based on the extrapolation of low-energy measurements. Up to the knee energies, typically three types of models were employed to fit the all particle spectrum. The first type is motivated by the diffusive shock acceleration or propagation process. In those models, the cutoff energies of CRs are expected to be proportional to the particle charge $Z$ [4, 6]. The second type is motivated by interaction processes, in which the cutoff or break energies are proportional to the atomic number $A$ [9 12 . The third type of the break is constant for all species. It is not well physically motivated, but might be a simple assumption [3]. Recent results show that the break energy of light components is lower than that of the allparticle knee, which disfavors this constant break energy scenario [15, 18 20]. Therefore, only the $Z$-dependent and $A$-dependent cases are considered in the following discussion.

To include the spectral hardenings at $\sim 200 \mathrm{GV}$, we parameterize the spectrum of each composition as

$$
\begin{aligned}
\frac{d \Phi^{i}}{d E}(E) & =\Phi_{0}^{i} \times\left(\frac{E}{E_{\mathrm{br}}}\right)^{-\gamma_{1}^{i}} \\
& \times\left(\frac{1+E / E_{\mathrm{br}}}{2}\right)^{\left[\gamma_{1}^{i}-\gamma_{2}^{i}+\beta \cdot \log \left(E / E_{\mathrm{had}}\right)\right]} \\
& \times e^{-E / E_{c}^{i}}
\end{aligned}
$$

where $E_{\mathrm{br}}$ is the break energy which describes the low energy (with a rigidity of a few GV) behavior of the spectrum, $\Phi_{0}^{i}$ is the absolute flux of the $i$ th element at $E_{\mathrm{br}}$, $\gamma_{1}^{i}\left(\gamma_{2}^{i}\right)$ is the spectral index below (above) $E_{\mathrm{br}}, E_{\mathrm{had}}$ is the energy characterizing the spectral hardening which is fixed to be $Z \cdot 200 \mathrm{GeV}$, and $\beta \cdot \log \left(E / E_{\mathrm{had}}\right)$ is an asymptotically hardening term used to describe the spectral hardening.

The proton and Helium spectra have been measured up to $\mathrm{TeV}$ by AMS-02 with very high precision [28, 29]. Their spectral parameters are fitted separately with Eq. (1). For the other major compositions, such as $\mathrm{C}, \mathrm{O}, \mathrm{Mg}, \mathrm{Al}, \mathrm{Si}$, and $\mathrm{Fe}$, the HEAO-3 data [43] are used to determine their spectral parameters. For simplicity, their low energy spectral parameters $\gamma_{1}$ and $R_{\mathrm{br}} \equiv E_{\mathrm{br}} / Z$ are assumed to be the same. For convenience, the spectral parameters of all species are tabulated in the Appendix.

To account for the spectra around the knee, we assume a $Z$ - or $A$-dependent cutoff of each species as

$$
E_{c}^{i}= \begin{cases}E_{c}^{p} \cdot Z, & \text { charge dependent } \\ E_{c}^{p} \cdot A, & \text { mass dependent }\end{cases}
$$

where $E_{c}^{p}$ is the cutoff energy of protons. Parameters $E_{c}^{p}$ correlates with $\beta$. They will be determined through fitting to the data.

\section{$2.2 \quad$ Fitting results}

The direct measurements of proton and Helium fluxes by AMS-02 [28, 29] and CREAM I+III [44], as well as the air shower array measurements of the light components $(\mathrm{H}+\mathrm{He})$ at high energies [15, 18, 19, 21] are used in the fits. The all-particle spectra are not included in the fits. We require that the calculated all-particle spectra are lower than the $2 \sigma$ upper bounds of the observations. Due to the uncertainties of the absolute energy calibration and the hadronic interaction models of the ground based CR experiments, the observed break energies of the knee of light components differ from each other.

We first classify the ground-based experiments into two groups, the Tibet group (including AS $\gamma$, ARGOYBJ, and WFCTA) and KASCADE. Together with the AMS-02 and CREAM data, we fit to each group of data using both the $Z$ - and $A$-dependent parameterizations of the knee. The best-fit parameters are given in Tables 1 and 2. The results show that the fit to AMS02+CREAM+KASCADE data gives a relatively large $\chi^{2}$ value, while the fit to AMS-02+CREAM+Tibet data is acceptable. This is due to that the KASCADE data favors a relatively high energy of the knee, which requires $\beta$ to be relatively small and the spectral hardening effect is not enough to match the CREAM data (see Fig. 1 for

\footnotetext{
* An anti-correlation between $\log \left(E_{p}\right)$ and $\beta$ is shown, which is basically due to a mathematical constraint. For a larger $\beta$, the spectra of individual compositions can not extend to very high energies without exceeding the all-particle spectra, and hence $\log \left(E_{p}\right)$ is required to be smaller.
} 

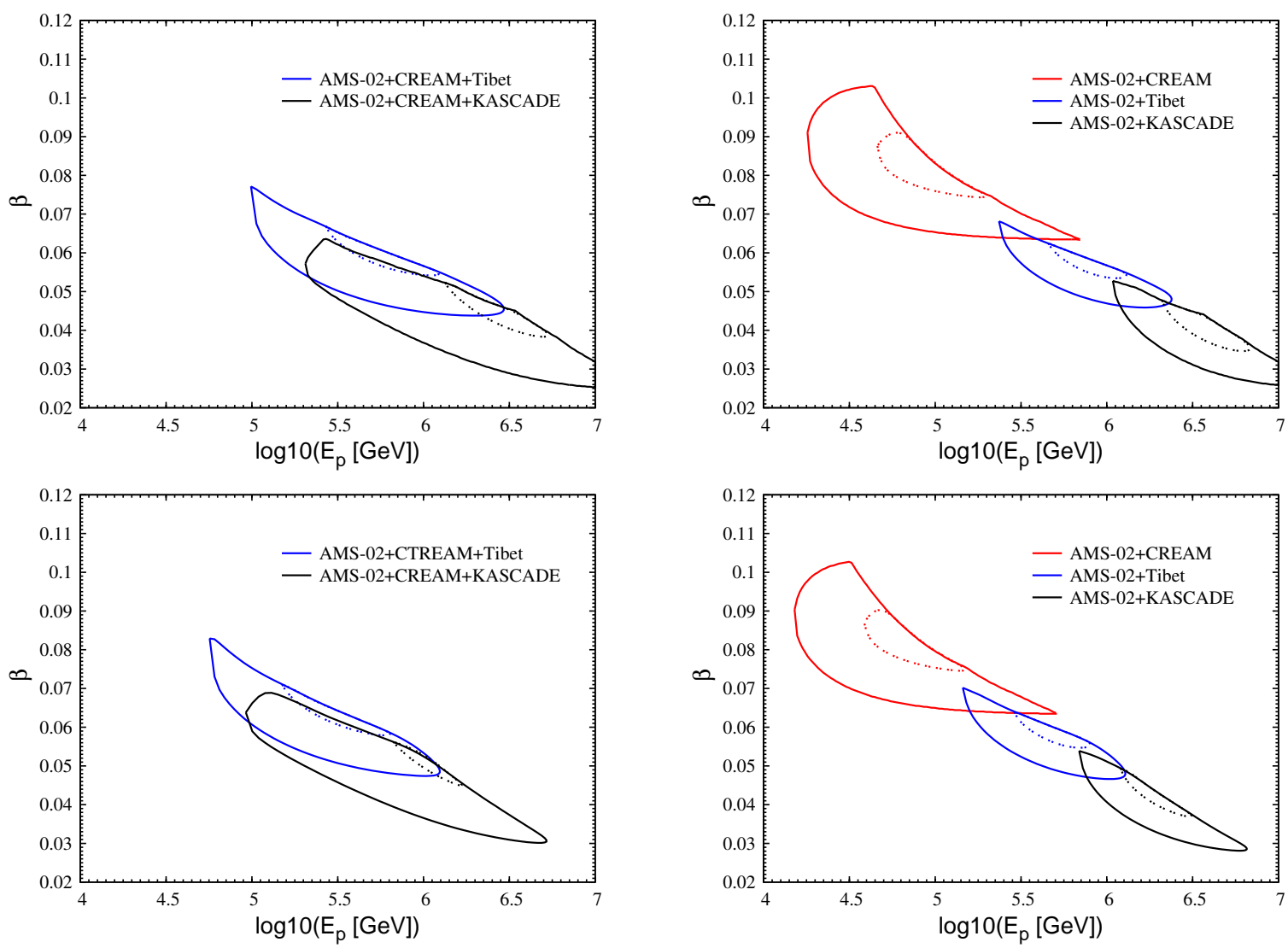

Fig. 1. The $68 \%$ (inner dashed) and $95 \%$ (outer solid) confidence regions of parameters $\beta$ and $E_{c}^{p}$. The left panels are for the combined fits to AMS-02+CREAM+Tibet and AMS-02+CREAM+KASCADE, and the right panels are for the fits to AMS-02+CREAM, AMS-02+Tibet, and AMS-02+KASCADE data, respectively. The upper panels are for Z-dependent and the lower panels are A-dependent cases.

Table 1. Low energy spectral parameters of protons and Helium nuclei of the "modified poly-gonato" model

\begin{tabular}{ccccccc}
\hline \hline Parameters & Species & AMS-02+CREAM+Tibet & AMS-02+CREAM+KASCADE & AMS-02+CREAM & AMS-02+Tibet & AMS-02+KASCADE \\
\hline$\Phi_{0}$ & $\mathrm{p}$ & 337.6 & 383.0 & 290.6 & 351.3 & 371.4 \\
$\left(\mathrm{~m}^{-2} \mathrm{~s}^{-1} \mathrm{Sr}^{-1} \mathrm{GeV}^{-1}\right)$ & $\mathrm{He}$ & 9.5 & 10.9 & 8.0 & 9.0 & 10.7 \\
\hline$R_{\mathrm{br}}$ & $\mathrm{p}$ & 2.15 & 1.94 & 2.41 & 2.07 & 1.99 \\
$(\mathrm{GV})$ & $\mathrm{He}$ & 3.36 & 2.94 & 4.11 & 3.82 & 3.41 \\
\hline$\gamma_{1}$ & $\mathrm{p}$ & -0.63 & -0.75 & -0.49 & -0.69 & -0.70 \\
& $\mathrm{He}$ & 0.07 & 0.00 & 0.15 & 0.12 & 0.02 \\
\hline$\gamma_{2}$ & $\mathrm{p}$ & 2.93 & 2.93 & 2.96 & 2.93 & 2.92 \\
& $\mathrm{He}$ & 2.84 & 2.83 & 2.86 & 2.84 & 2.82 \\
\hline \hline
\end{tabular}

the contours of parameters $\log \left(E_{p}\right)$ and $\left.\beta^{*}\right)$. To compare with the standard poly-gonato model, we perform the fittings with $\beta=0$. The minimum $\chi^{2}$ values of these fittings are given in Table 2. It is obvious that these fittings are much worse than the "modified poly-gonato" model.

Motivated by the facts that the CREAM data might reveal hint of spectral softening above $\sim 20 \mathrm{TeV}[44]$ and the combined fit of AMS-02+CREAM+KASCADE does not give a good enough fit, we separate the CREAM data from the ground-based measurements and re-do the fits with AMS-02+CREAM, AMS-02+Tibet, and AMS02+KASCADE data, respectively. The favored confidence regions of parameters $\log \left(E_{p}\right)$ and $\beta$ are shown in 
Chinese Physics C Vol. XX, No. X (201X) XXXXXX

Table 2. High energy spectral parameters of the "modified poly-gonato" model

\begin{tabular}{ccccccc}
\hline \hline Parameters & Mode & \multirow{2}{*}{ AMS-02+CREAM+Tibet } & AMS-02+CREAM+KASCADE & AMS-02+CREAM & AMS-02+Tibet & AMS-02+KASCADE \\
\hline$\beta$ & $Z$ & 0.063 & 0.045 & 0.083 & 0.062 & 0.041 \\
& $A$ & 0.068 & 0.051 & 0.083 & 0.065 & 0.048 \\
\hline$E_{c}^{p}$ & $Z$ & $5.7 \times 10^{5}$ & $2.9 \times 10^{6}$ & $1.4 \times 10^{5}$ & $7.4 \times 10^{5}$ & $3.7 \times 10^{6}$ \\
$(\mathrm{GeV})$ & $A$ & $2.9 \times 10^{5}$ & $1.3 \times 10^{6}$ & $1.3 \times 10^{5}$ & $4.1 \times 10^{5}$ & $1.7 \times 10^{6}$ \\
\hline$\chi^{2} /$ dof & $Z$ & $128.5 / 195$ & $225.0 / 171$ & $68.7 / 154$ & $63.4 / 171$ & $76.4 / 147$ \\
$\chi^{2} /$ dof & $A$ & $116.7 / 195$ & $191.1 / 171$ & $68.5 / 154$ & $71.7 / 171$ & $71.6 / 147$ \\
\hline$\beta=0$ & & & & & & $399.9 / 148$ \\
\hline$\chi^{2} /$ dof & $Z$ & $951.2 / 196$ & $1042.8 / 172$ & $678.4 / 155$ & $653.2 / 172$ & $399.8 / 148$ \\
$\chi^{2} /$ dof & $A$ & $949.4 / 196$ & $1001.6 / 172$ & $678.2 / 155$ & $651.7 / 172$ & 397 \\
\hline \hline
\end{tabular}

the right panel of Fig. 1, It is shown that the AMS$02+$ CREAM fit tends to favor a relatively low break energy of $E_{p}$ compared with the other two fits. The parameter regions of AMS-02+Tibet and AMS-02+CREAM (AMS-02+KASCADE) overlap with each other at the 95\% confidence level. However, the results of AMS02+CREAM and AMS-02+KASCADE do not overlap. Fig. 2] shows the best-fit results of fluxes of several major components in CRs for the $Z$-dependent scenario, compared with the data. In this figure, panels (a)-(c) are the spectra of protons, Helium, and $\mathrm{H}+\mathrm{He}$ for the three groups of fits (AMS-02+CREAM, AMS-02+Tibet, and AMS-02+KASCADE). Panel (d) is for $\mathrm{C}$ and O, panel (e) is for $\mathrm{Mg}, \mathrm{Al}$, and $\mathrm{Si}$, panel (f) is for $\mathrm{Fe}$, respectively. Panels (g)-(i) are the all-particle spectra of the three groups.

We find that for the AMS-02+CREAM fit, the favored energy of the knee of light components is relatively low, which under-shoots the all-particle spectra. However, given the large uncertainties and limited coverage of the energy range of the CREAM data, the constraint on the cutoff energy is very loose (see Fig. 1). The Tibet experimental data gives a median knee energy of light components and is consistent with the all-particle spectra below tens of $\mathrm{PeV}$. The KASCADE data gives the highest energy of the knee, which slightly over-shoots, but is roughly consistent with, the all-particle spectra.

The results for the $A$-dependent scenario are shown in Fig. 3. We have similar conclusion with that of the $Z$-dependent scenario. For the $A$-dependent case, the knee energy of protons is smaller by a factor of $\sim 2$ compared with that of the $Z$-dependent case. At present it is difficult to distinguish these two cases, and we need measurements of the knee of either protons or Helium to distinguish them.

\section{The spatially-dependent diffusion model}

In the above section, we introduce a "modified polygonato" model to reproduce the wide-band spectra of CRs. One possible physical explanation of the spectral hardening $(\beta \cdot \log E)$ is the spatially-dependent diffusion of particles [36, 39, 41, 42, 50]. In a simplified version, i.e., CRs diffuse separately in the disk region and halo region (the two-halo model), the hardening of the primary CR nuclei and the excesses of secondary particles can be reasonably accounted for $[36,41,42,50$. . Here we extrapolate this model to the knee region to reproduce the results of the phenomenological "modified poly-gonato" model.

\subsection{Model description}

We employ the diffusion reacceleration model to describe the propagation of CR particles [see e.g., 51, 52]. A cylindrical geometry is assumed. The propagation is confined in a halo with half height of $z_{h}$. The diffusion coefficient, $D_{x x}$, depends on both the spatial coordinates $(r, z)$ and the particle rigidity, which is parameterized as 41]

$$
D_{x x}(r, z, \rho)=\left\{\begin{array}{lll}
\eta(r, z) \beta\left(\frac{\rho}{\rho_{0}}\right)^{\varepsilon(r, z)}, & |z|<\xi z_{h} & \text { (disk) } \\
D_{0} \beta\left(\frac{\rho}{\rho_{0}}\right)^{\delta_{0}}, & |z|>\xi z_{h} & \text { (halo) }
\end{array}\right.
$$

where $\beta$ is the velocity of the particle in unit of light velocity $c, D_{0}$ represents the normalization of the halo diffusion efficient at $\rho_{0}=4 \mathrm{GV}, \delta_{0}$ characterizes the rigidity dependence of the diffusion coefficient, $\xi z_{h}$ denotes the thickness of the disk, $\eta(r, z)$ and $\varepsilon(r, z)$ describes the spatial dependence of the diffusion coefficient in the disk. $\eta(r, z)$ and $\varepsilon(r, z)$ can be related to the source distribution $f(r)$, via a unified form as [4],

$F(r, z)= \begin{cases}\left(1 /\left[1+e^{f(r)}\right]-A_{i}\right)\left[1-\left(z / \xi z_{h}\right)^{4}\right]+F_{0} \cdot\left(z / \xi z_{h}\right)^{4} & \text { (disk) } \\ F_{0} & \text { (halo) }\end{cases}$ 

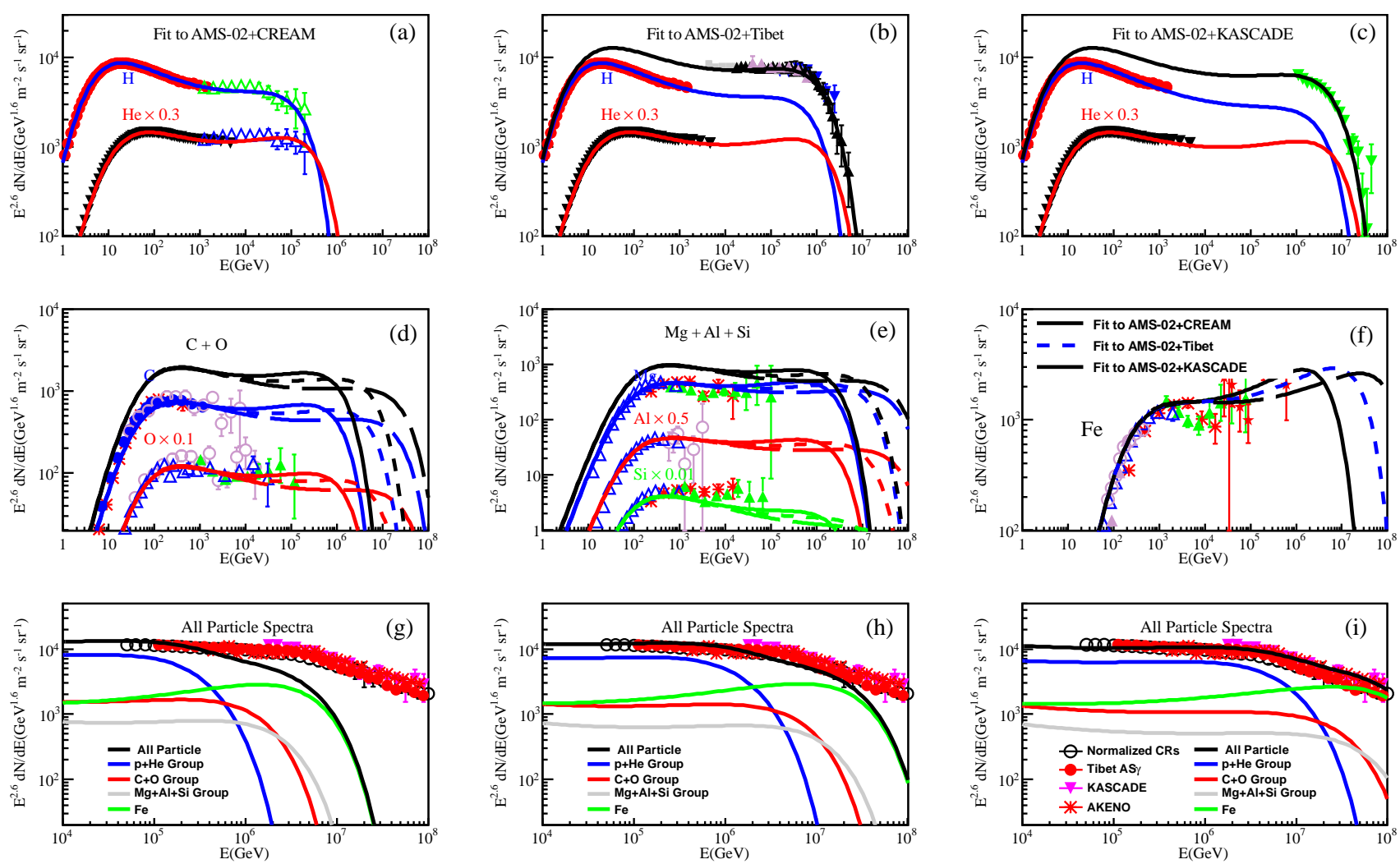

Fig. 2. The comparison between the best-fit results and the experimental data, for the $Z$-dependent case. The proton data are from: AMS-02 28], CREAM [44], ATIC-2 25]; the Helium data are from: AMS-02 29], CREAM 44]; ATIC-2 25]; the Carbon, Oxygen, Magnesium, Aluminium, Silicon, and Iron data are from: HEAO-3 43], TRACER 45], ATIC-2 46] and CREAM-II [7]; the proton + Helium data are from: KASCADE 17], Tibet-AS $\gamma$ 15], WFCTA [19], ARGO-YBJ 18, 20]; the all-particle data are from: Tibet-AS $\gamma$ [48], KASCADE [17], Akeno [49], and the normalized average one [3].

where $A_{i}$ is a constant with $i$ denoting $\eta$ or $\varepsilon, F_{0}$ is the $D_{0}$ and $\delta_{0}$.

The reacceleration is described by a diffusion in the momentum space, with a relation between $D_{\mathrm{pp}}$ and $D_{\mathrm{xx}}$ as [53]

$$
D_{p p} D_{x x}=\frac{4 p^{2} v_{A}^{2}}{3 \delta\left(4-\delta^{2}\right)(4-\delta) w},
$$

where $p$ is the momentum of a particle, $\delta$ is the power-law index of the rigidity dependence of the spatial diffusion coefficient (see Eq. (3) ), $v_{A}$ is the Alfven speed, $w$ is the ratio of the magnetohydrodynamic wave energy density to the magnetic field energy density which is assumed to be 1 .

The injection spectrum of CR nuclei is assumed to be broken power-law with an exponential cutoff

$$
q_{\mathrm{inj}}(E)=q_{0} e^{-E / E_{c}^{i}} \times\left\{\begin{array}{ll}
\left(E / E_{\mathrm{br}}\right)^{-\gamma_{1}}, & E<E_{\mathrm{br}} \\
\left(E / E_{\mathrm{br}}\right)^{-\gamma_{2}}, & E \geq E_{\mathrm{br}}
\end{array} .\right.
$$

where $q_{0}$ is the normalization factor, $E_{\mathrm{br}}$ is the break energy, $\gamma_{1}, \gamma_{2}$ are the spectral indices below and above $E_{\mathrm{br}}$, and $E_{c}^{i}$ characterizes the spectral cutoff around the knee. The relative abundances of different nuclei are adopted as the default values used in DRAGON [54]. Similar as in Sec. 2, we consider either the $Z$-dependent and $A$-dependent models of the cutoff.

Low energy particles $(E \lesssim 10 \mathrm{GeV} / \mathrm{n})$ will be modulated by solar activities, showing suppress of their low energy fluxes. We use the force-field approximation to account for this solar modulation 55]. In this work, The modulation potential $\Phi$ is fixed to be $550 \mathrm{MV}$ for all the nuclei except for $\mathrm{B} / \mathrm{C}$ whose modulation potential is adopted as $200 \mathrm{MV}$.

\subsection{Results}

\subsubsection{Primaries}

We use the numerical code DRAGON to calculate the spatial dependent diffusion of CRs [54]. The injection spectral parameters are given in Tables 3 . The parameter $\gamma_{2}$ differs for each species. They are tuned to fit the data for the major compositions. And for the less abundant nuclei, we assume the same difference of $\gamma_{2}$ from 

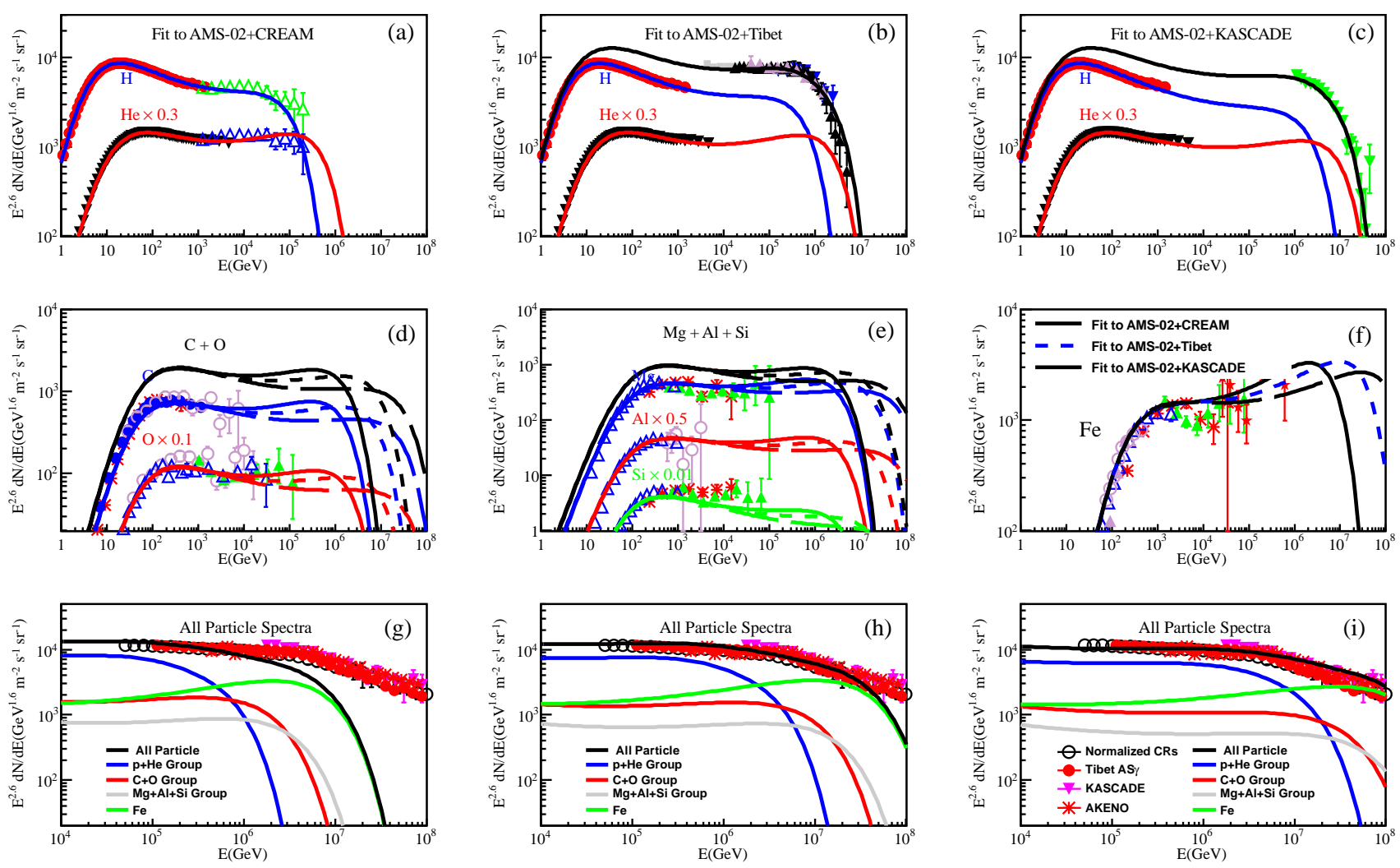

Fig. 3. Same as Fig. 2 but for the $A$-dependent model.

that of protons as in Sec. 2. The full compilation of $\gamma_{2}$ are given in the Appendix.

The propagation parameters are given in Table 4. Note that, in principle, the allowed parameter space needs to be estimated by a global fitting to the data, which is CPU-time consuming and is left for future studies. We compare the model predictions to the three data sets of the knee of the light components, as described in Sec. 2. Results of the primary CRs are shown in Figs. 4 and 5. for the $Z$ - and $A$-dependent cutoff scenarios of the knee. We find that the results are very similar to that of the "modified poly-gonato" model. It is shown that a log-parabolic shape of the energy spectrum is a good approximation of a class of models with smooth hardenings.

\subsubsection{Secondaries}

Secondary particles will be produced by collisions of primary CRs with the interstellar medium when they propagate in the Galaxy. It is believed that most of antiprotons and Borons are such secondaries, which can be very effective to probe the particle propagation process. We calculate the expected $\bar{p} / p$ and $\mathrm{B} / \mathrm{C}$ ratios of this spatially-dependent diffusion model, as shown in Fig. 6. These results are reasonably consistent with the observational data. However, we do find that the second-toprimary ratio becomes asymptotically flatter at high en- ergies, which is different from the simple uniform diffusion scenario. This can be tested with future observations of the $\mathrm{B} / \mathrm{C}$ ratio to higher energies. 
Chinese Physics C Vol. XX, No. X (201X) XXXXXX

Table 3. Injection parameters of the "spatially-dependent diffusion" model

\begin{tabular}{ccccc}
\hline \hline & mode & AMS-02+CREAM & AMS-02+Tibet & AMS-02+KASCADE \\
\hline$E_{b r}(\mathrm{GV})$ & & 9.5 & 9.5 & 9.5 \\
$\gamma_{1}$ & & 1.85 & 1.85 & 1.85 \\
$E_{c}^{p}(\mathrm{GeV})$ & $Z$ & $1.8 \times 10^{5}$ & $1.1 \times 10^{6}$ & $3.9 \times 10^{6}$ \\
& $A$ & $1.5 \times 10^{5}$ & $6.6 \times 10^{5}$ & $2.0 \times 10^{6}$ \\
\hline \hline
\end{tabular}

Table 4. Propagation parameters of the "spatially-dependent diffusion" model

\begin{tabular}{cccc}
\hline \hline & AMS-02+CREAM & AMS-02+Tibet & AMS-02+KASCADE \\
\hline$D_{0}\left(\mathrm{~cm}^{2} / \mathrm{s}\right)$ & $6.8 \times 10^{28}$ & $6.8 \times 10^{28}$ & $6.8 \times 10^{28}$ \\
$\delta_{0}$ & 0.58 & 0.52 & 0.5 \\
$v_{A}(\mathrm{~km} / \mathrm{s})$ & 16 & 16 & 16 \\
$z_{h}(\mathrm{kpc})$ & 5 & 5 & 5 \\
$\xi$ & 0.14 & 0.12 & 0.11 \\
$A_{\eta}$ & 0.10 & 0.10 & 0.10 \\
$A_{\varepsilon}$ & -0.17 & -0.16 & -0.14 \\
\hline \hline
\end{tabular}
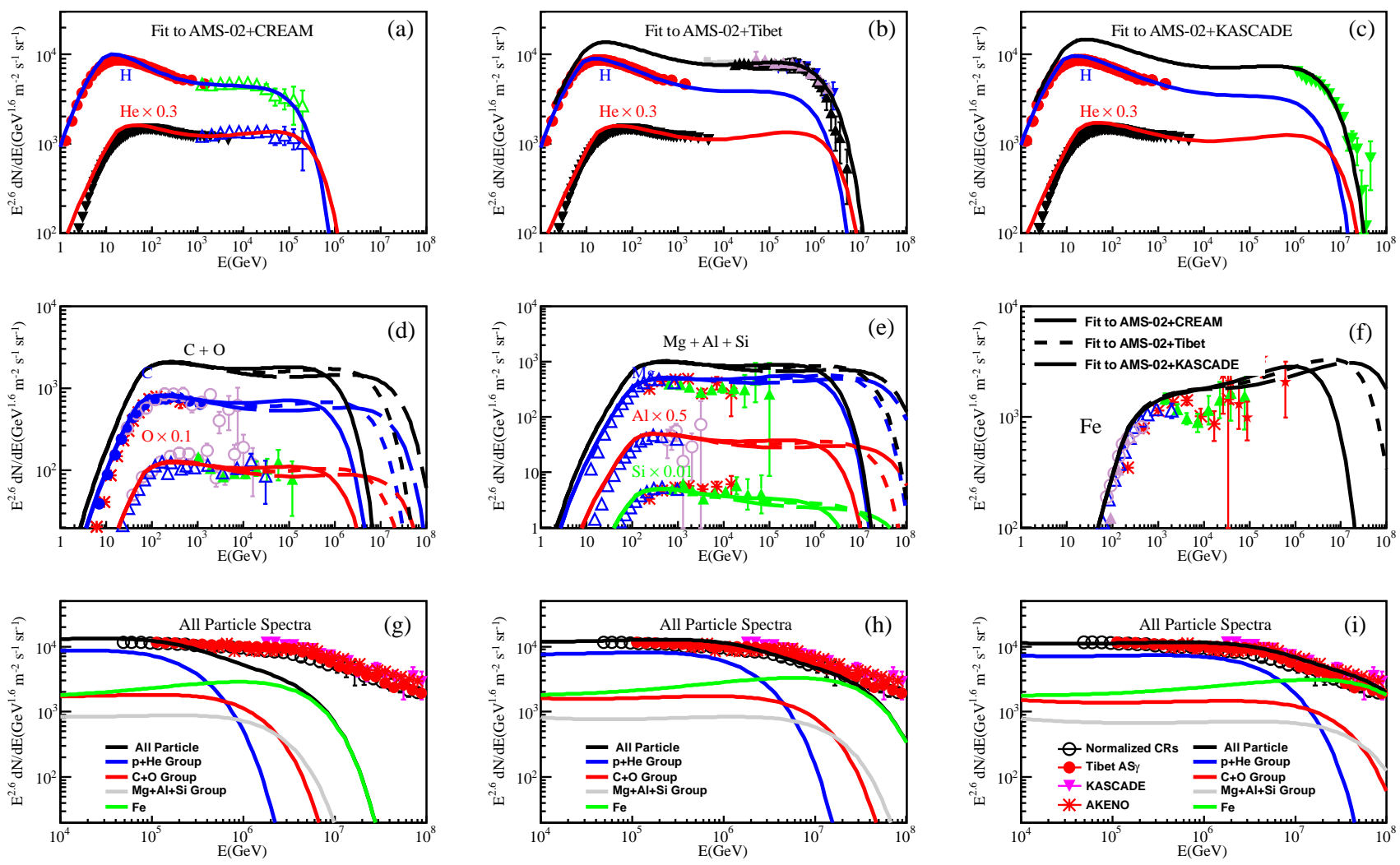

Fig. 4. Same as Fig. 2, but for the spatially-dependent diffusion model.

\subsubsection{Anisotropy}

The flow of CRs will form a dipole anisotropy of arrival directions when observed at a fixed point. We cal- culate the amplitude of the dipole anisotropy of CRs as

$$
A=\frac{D}{c} \frac{\nabla \phi}{\phi}
$$



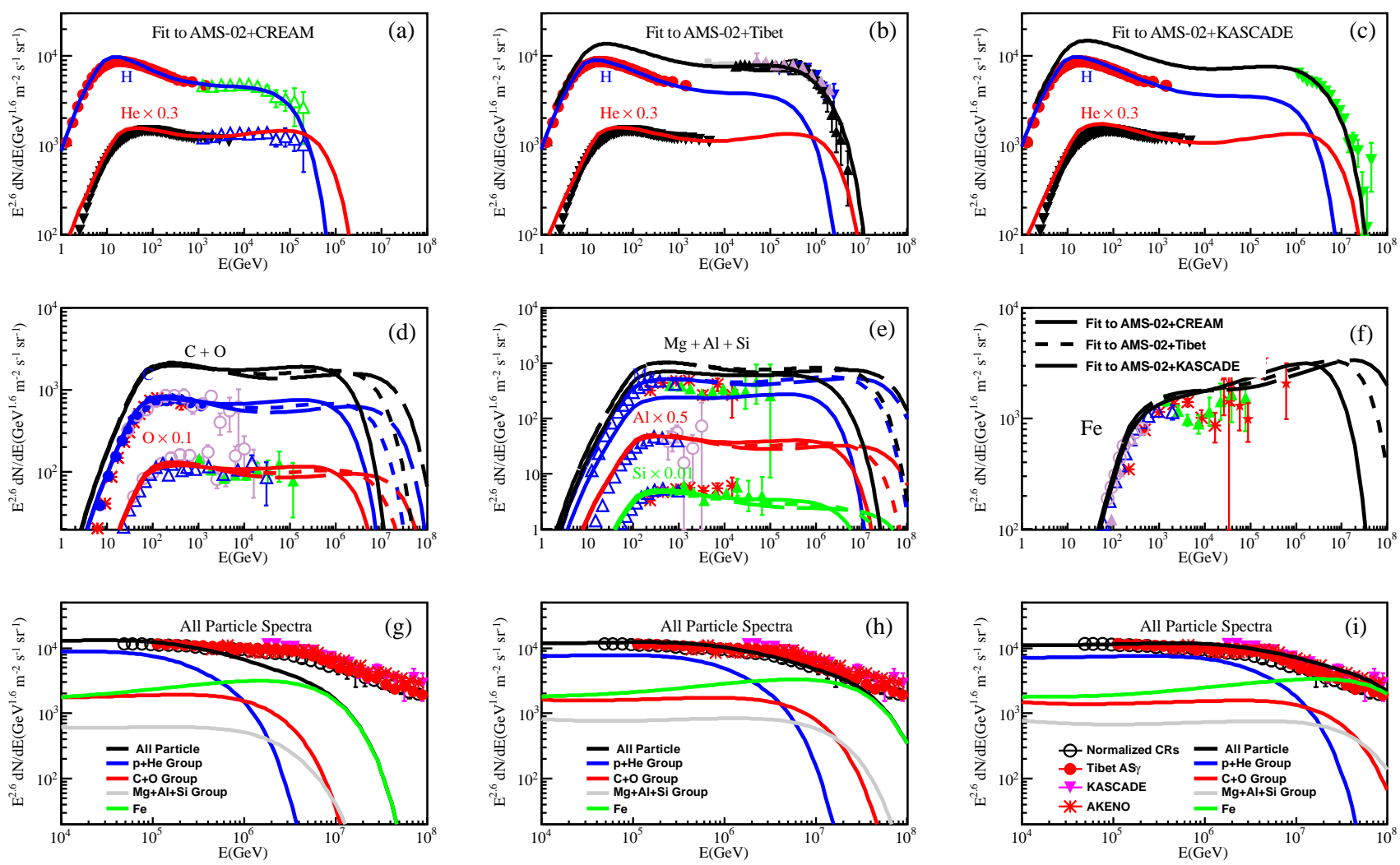

Fig. 5. Same as Fig. 3, but for the spatially-dependent diffusion model.
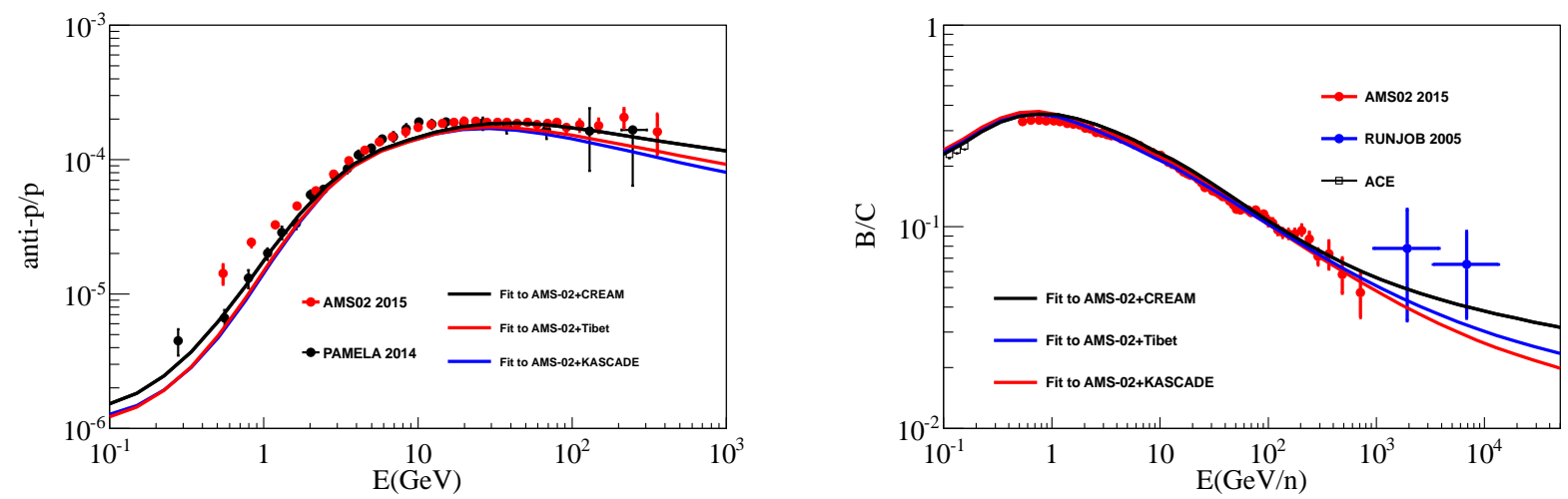

Fig. 6. The calculated $\bar{p} / p$ (left panel) and $\mathrm{B} / \mathrm{C}$ (right panel) ratios of the spatially-dependent diffusion model. The $\bar{p} / p$ data are from AMS-02 [56] and PAMELA [57]; the B/C data are from AMS-02 [56], ACE [58] and RUNJOB [59].

where $\phi$ is the locally observed differential fluxes of CRs. The dipole anisotropy amplitude as a function of energy is given in Fig. 7 The amplitude of the anisotropy is smaller than the prediction of the standard diffusion model [36, 41, 42, 50], and is consistent with observations up to a few tens of $\mathrm{TeV}$. Note, however, the phase of the observed anisotropy shows an evolution with energy, which can not be simply accounted for by the diffusion process [60]. More complicated process like the effect of the local magnetic field and/or local sources may be responsible for it [16].

\section{Conclusion and discussion}

Recent observations revealed new features on the CR spectra, including spectral hardenings at $\sim 200 \mathrm{GV}$ rigidities from balloon or space detectors and the knee of light components ( $\mathrm{p}$ and $\mathrm{He}$ ) from air shower experiments. In this work we develop a modified version of the "poly-gonato" model of the knee, taking into account 


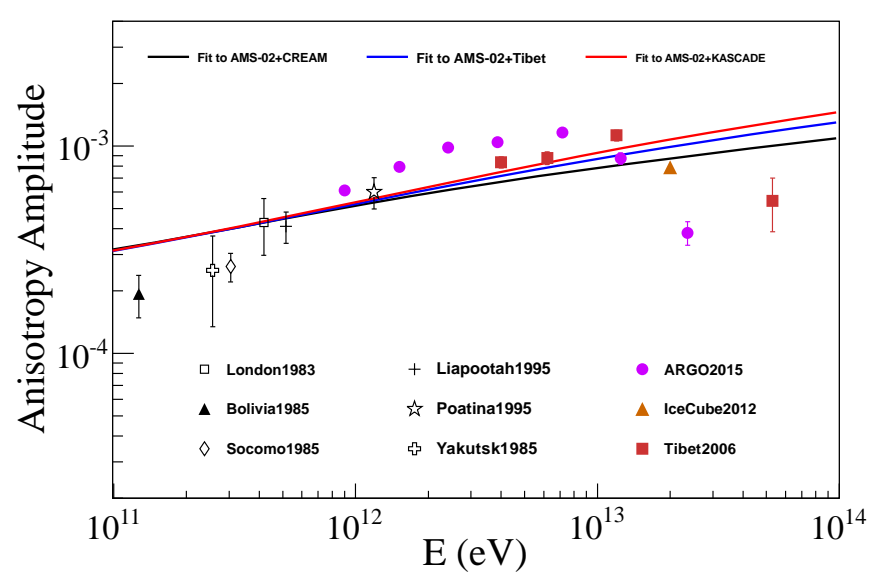

Fig. 7. The calculated anisotropy of CRs for the spatially-dependent diffusion model. The data are from underground muon observations: London1983 61], Bolivia1985 62], Socorro1985 [62], Yakutsk1985 [62], Liapootah1995 63], Poatina1995 64], and air shower array experiments: Tibet2006 [65], IceCube2012 [66], ARGO2015 [67].

such new data. A log-parabolic term of the spectrum is employed to describe the spectral hardenings. As for the knee, we adopt an exponential cutoff spectrum to describe it, with the cutoff energy being proportional to $Z$ or $A$ of each species. We then fit the spectral parameters to the observational data. Due to the difficulty of the absolute energy calibration in the air shower experiments, the break positions of the light component spectra differ to some degree among different experiments. Therefore the fits are done for different datasets separately, based on the light component measurements by CREAM, Tibet experiments (AS $\gamma$, WFCTA and ARGOYBJ), and KASCADE. We also try to jointly fit the CREAM data and the air shower experimental data, and find that the fitting goodness is relatively poor, expecially for CREAM+KASCADE. In all the fits, the AMS02 measurements at low energies $(\lesssim \mathrm{TeV})$ are included.

The results show that the knee energies inferred from different data groups are marginally consistent with each other. The CREAM data slightly favors a relatively low energy knee of protons and Helium nuclei, which undershoots the all-particle spectra individual fits. In this case, an extra component of CRs below the knee may be required [e.g., 24, 68]. The Tibet experiment and KASCADE data of light components are roughly consistent with the all-particle data.

There are no good measurements of CR spectra in the energy range of $1-100 \mathrm{TeV}$. For example, the proton and Helium spectra by CREAM [44] differ much from that by ATIC-2 25]. A direct comparison of the AMS-02 measured fluxes of Helium and that by CREAM shows that the CREAM ones are higher by about $20 \%$ at $\mathrm{TeV} /$ nucleon [29]. Further more precise measurements of the energy spectra of various species, by e.g., CALET [69], DAMPE [70], and LHAASO 71] will be very im- portant to better determine the model parameters.

It is also possible that the fitting function, which basically has smooth behaviors of the hardening and cutoff, is not good enough to describe the data. If there are some sharp structures of the spectra, the CREAM proton and Helium spectra and the all-particle data might be better consistent with each other. However, in such a case the model may need fine tuning.

Finally we give a physical model with spatiallydependent diffusion of CRs to reproduce the results of this phenomenological "modified poly-gonato" model. Apart from the potential inconsistencies among different data sets, the energy spectra of the primary CRs, the secondary-to-primary ratios, and the amplitude of the anisotropy are shown to be consistent with observations. This model predicts asymptotical hardening of the $\mathrm{B} / \mathrm{C}$ ratio above hundreds of $\mathrm{TeV} / \mathrm{n}$, which can also be tested with future measurements.

\section{Acknowledgments}

This work is supported by the National Key Research and Development Program of China (No. 2016YFA0400200), the National Natural Science Foundation of China (Nos. 11635011, 11761141001, 11663006, 11722328), and the 100 Talents program of Chinese Academy of Sciences.

\section{References}

1 G. V. Kulikov and G. B. Kristiansen. J. Exp. Theor. Phys., 35:635, 1958.

2 J. R. Hörandel. Models of the knee in the energy spectrum of cosmic rays. Astroparticle Physics, 21:241-265, June 2004.

3 J. R. Hörandel. On the knee in the energy spectrum of cosmic rays. Astroparticle Physics, 19:193-220, May 2003.

4 P. O. Lagage and C. J. Cesarsky. The maximum energy of cosmic rays accelerated by supernova shocks. Astron. Astrophys. , 125:249-257, September 1983. 
5 H. J. Voelk and P. L. Biermann. Maximum energy of cosmicray particles accelerated by supernova remnant shocks in stellar wind cavities. Astrophys. J. Lett. , 333:L65-L68, October 1988.

6 V. S. Ptuskin, S. I. Rogovaya, V. N. Zirakashvili, et al. Diffusion and drift of very high energy cosmic rays in galactic magnetic fields. Astron. Astrophys. , 268:726-735, February 1993.

7 E. G. Berezhko. Maximum energy of cosmic rays accelerated by supernova shocks. Astroparticle Physics, 5:367-378, October 1996.

8 B. Wiebel-Sooth, P. L. Biermann, and H. Meyer. Cosmic rays. VII. Individual element spectra: prediction and data. Astron. Astrophys. , 330:389-398, February 1998.

9 S. Karakula and W. Tkaczyk. The formation of the cosmic ray energy spectrum by a photon field. Astroparticle Physics, 1:229-237, March 1993.

10 J. Candia, L. N. Epele, and E. Roulet. Cosmic ray photodisintegration and the knee of the spectrum. Astroparticle Physics, 17:23-33, April 2002.

11 D. Kazanas and A. Nicolaidis. Cosmic Ray "Knee": A Herald of New Physics? International Cosmic Ray Conference, 5:1760, August 2001.

12 H.-B. Hu, Q. Yuan, B. Wang, et al. On the $\mathrm{e}^{+} \mathrm{e}^{-}$Excesses and the Knee of the Cosmic Ray Spectra Hints of Cosmic Ray Acceleration in Young Supernova Remnants. Astrophys. J. Lett. , 700:L170-L173, August 2009.

13 B. Wang, Q. Yuan, C. Fan, et al. A study on the sharp knee and fine structures of cosmic ray spectra. Science China Physics, Mechanics, and Astronomy, 53:842-847, May 2010.

14 Y.-Q. Guo, Z.-Y. Feng, Q. Yuan, C. Liu, and H.-B. Hu. On the Galactic Center being the main source of galactic cosmic rays as evidenced by recent cosmic ray and gamma ray observations. New Journal of Physics, 15(1):013053, January 2013.

15 J. Huang. Primary proton and helium spectra at energy range from $50 \mathrm{TeV}$ to $1 \mathrm{PeV}$ observed with (YAC+Tibet-III ) hybrid experiment. International Cosmic Ray Conference, July 2013.

16 M. Ahlers. Deciphering the Dipole Anisotropy of Galactic Cosmic Rays. Physical Review Letters, 117(15):151103, October 2016.

17 W. D. Apel, J. C. Arteaga-Velázquez, K. Bekk, et al. KASCADE-Grande measurements of energy spectra for elemental groups of cosmic rays. Astroparticle Physics, 47:54-66, July 2013.

18 B. Bartoli, P. Bernardini, X. J. Bi, et al. Cosmic ray proton plus helium energy spectrum measured by the ARGO-YBJ experiment in the energy range $3-300 \mathrm{TeV}$. Phys. Rev. D, 91(11):112017, June 2015.

19 B. Bartoli, P. Bernardini, X. J. Bi, et al. Knee of the cosmic hydrogen and helium spectrum below $1 \mathrm{PeV}$ measured by ARGO-YBJ and a Cherenkov telescope of LHAASO. Phys. Rev. D , 92(9):092005, November 2015.

20 A. D'Amone, I. De Mitri, and A. Surdo. Measurement of the cosmic ray all-particle and light-component energy spectra with the ARGO-YBJ experiment. ArXiv e-prints, February 2015.

21 P. Montini and S. M. Mari. The bending of the proton plus helium flux in primary cosmic rays measured by the ARGO-YBJ experiment in the energy range from $20 \mathrm{TeV}$ to $5 \mathrm{PeV}$. ArXiv e-prints, August 2016.

22 M. Shibata, Y. Katayose, J. Huang, and D. Chen. Chemical Composition and Maximum Energy of Galactic Cosmic Rays. Astrophys. J. , 716:1076-1083, June 2010.

23 Y. Zhao, H.-Y. Jia, and F.-R. Zhu. Reconciling the light component and all-particle cosmic ray energy spectra at the knee. Chinese Physics C, 39(12):125001, December 2015.

24 S. Thoudam, J. P. Rachen, A. van Vliet, et al. Cosmic-ray energy spectrum and composition up to the ankle: the case for a second Galactic component. Astron. Astrophys. , 595:A33, October 2016.

25 A. D. Panov, J. H. Adams, Jr., H. S. Ahn, et al. Elemental energy spectra of cosmic rays from the data of the ATIC-2 experiment. Bulletin of the Russian Academy of Science, Phys., 71:494-497, April 2007.

26 H. S. Ahn, P. Allison, M. G. Bagliesi, et al. Discrepant Hardening Observed in Cosmic-ray Elemental Spectra. Astrophys. J. Lett. , 714:L89-L93, May 2010.

27 O. Adriani, G. C. Barbarino, G. A. Bazilevskaya, et al. PAMELA Measurements of Cosmic-Ray Proton and Helium Spectra. Science, 332:69-, April 2011.

28 M. Aguilar, D. Aisa, B. Alpat, et al. Precision Measurement of the Proton Flux in Primary Cosmic Rays from Rigidity 1 GV to 1.8 TV with the Alpha Magnetic Spectrometer on the International Space Station. Physical Review Letters, 114(17):171103, May 2015.

29 M. Aguilar, D. Aisa, B. Alpat, et al. Precision Measurement of the Helium Flux in Primary Cosmic Rays of Rigidities 1.9 GV to $3 \mathrm{TV}$ with the Alpha Magnetic Spectrometer on the International Space Station. Physical Review Letters, 115(21):211101, November 2015.

30 V. I. Zatsepin and N. V. Sokolskaya. Three component model of cosmic ray spectra from $10 \mathrm{GeV}$ to $100 \mathrm{PeV}$. Astron. Astrophys. , 458:1-5, October 2006.

31 Q. Yuan, B. Zhang, and X.-J. Bi. Cosmic ray spectral hardening due to dispersion in the source injection spectra. Phys. Rev. D , 84(4):043002, August 2011.

32 S. Thoudam and J. R. Hörandel. Nearby supernova remnants and the cosmic ray spectral hardening at high energies. Mon. Not. Roy. Astron. Soc., 421:1209-1214, April 2012.

33 P. L. Biermann, J. K. Becker, J. Dreyer, et al. The Origin of Cosmic Rays: Explosions of Massive Stars with Magnetic Winds and Their Supernova Mechanism. Astrophys. J. , 725:184-187, December 2010.

34 V. Ptuskin, V. Zirakashvili, and E.-S. Seo. Spectra of CosmicRay Protons and Helium Produced in Supernova Remnants. Astrophys. J. , 763:47, January 2013.

35 S. Thoudam and J. R. Hörandel. GeV-TeV cosmic-ray spectral anomaly as due to reacceleration by weak shocks in the Galaxy. Astron. Astrophys., 567:A33, July 2014.

36 N. Tomassetti. Origin of the Cosmic-Ray Spectral Hardening. Astrophys. J. Lett., 752:L13, June 2012.

37 D. Gaggero, D. Grasso, A. Marinelli, A. Urbano, and M. Valli. The Gamma-Ray and Neutrino Sky: A Consistent Picture of Fermi-LAT, Milagro, and IceCube Results. Astrophys. J. Lett. , 815:L25, December 2015.

38 N. Tomassetti. Cosmic-ray protons, nuclei, electrons, and antiparticles under a two-halo scenario of diffusive propagation. Phys. Rev. D , 92(8):081301, October 2015.

39 C. Jin, Y.-Q. Guo, and H.-B. Hu. Spatial dependent diffusion of cosmic rays and the excess of primary electrons derived from high precision measurements by AMS-02. Chinese Physics $C$, 40(1):015101, January 2016.

40 Y. Q. Guo, H. B. Hu, and Z. Tian. On the Contribution of "Fresh" Cosmic Rays to the Excesses of Secondary Particles. ArXiv e-prints, December 2014.

41 Y.-Q. Guo, Z. Tian, and C. Jin. Spatial-dependent Propagation of Cosmic Rays Results in the Spectrum of Proton, Ratios of $\mathrm{P} / \mathrm{P}$, and $\mathrm{B} / \mathrm{C}$, and Anisotropy of Nuclei. Astrophys. J. , 819:54, March 2016.

42 J. Feng, N. Tomassetti, and A. Oliva. Bayesian analysis of spatial-dependent cosmic-ray propagation: Astrophysical background of antiprotons and positrons. Phys. Rev. D, 94(12):123007, December 2016.

43 J. J. Engelmann, P. Ferrando, A. Soutoul, P. Goret, and E. Juliusson. Charge composition and energy spectra of cosmicray nuclei for elements from Be to NI - Results from HEAO-3C2. Astron. Astrophys., 233:96-111, July 1990.

44 Y. S. Yoon, T. Anderson, A. Barrau, et al. Proton and Helium Spectra from the CREAM-III Flight. Astrophys. J. , 839:5, 
April 2017.

45 M. Ave, P. J. Boyle, F. Gahbauer, et al. Composition of Primary Cosmic-Ray Nuclei at High Energies. Astrophys. J. , 678:262-273, May 2008.

46 A. D. Panov, J. H. Adams, H. S. Ahn, et al. Energy spectra of abundant nuclei of primary cosmic rays from the data of ATIC2 experiment: Final results. Bulletin of the Russian Academy of Sciences, Physics, 73:564-567, June 2009.

47 H. S. Ahn, P. Allison, M. G. Bagliesi, et al. Energy Spectra of Cosmic-ray Nuclei at High Energies. Astrophys. J. , 707:593603, December 2009.

48 M. Amenomori, X. J. Bi, D. Chen, et al. The All-Particle Spectrum of Primary Cosmic Rays in the Wide Energy Range from $10^{14}$ to $10^{17} \mathrm{eV}$ Observed with the Tibet-III Air-Shower Array. Astrophys. J. , 678:1165-1179, May 2008.

49 M. Nagano, T. Hara, Y. Hatano, et al. Energy spectrum of primary cosmic rays between $10^{14.5}$ and $10^{18} \mathrm{eV}$. Journal of Physics G Nuclear Physics, 10:1295-1310, September 1984.

$50 \mathrm{~N}$. Tomassetti. Origin of the spectral upturn in the cosmic-ray C /Fe and O /Fe ratios. Phys. Rev. D , 92(6):063001, September 2015 .

51 A. W. Strong, I. V. Moskalenko, and V. S. Ptuskin. Cosmic-Ray Propagation and Interactions in the Galaxy. Annual Review of Nuclear and Particle Science, 57:285-327, November 2007.

52 Q. Yuan, S.-J. Lin, K. Fang, and X.-J. Bi. Propagation of cosmic rays in the AMS-02 era. Phys. Rev. D , 95(8):083007, April 2017.

53 E. S. Seo and V. S. Ptuskin. Stochastic reacceleration of cosmic rays in the interstellar medium. Astrophys. J. , 431:705-714, August 1994.

54 C. Evoli, D. Gaggero, D. Grasso, and L. Maccione. Cosmic ray nuclei, antiprotons and gamma rays in the galaxy: a new diffusion model. Journal of Cosmology and Astroparticle Physics, 10:18, October 2008.

55 L. J. Gleeson and W. I. Axford. Solar Modulation of Galactic Cosmic Rays. Astrophys. J. , 154:1011, December 1968.

56 AMS-02 collaboration. Talks at the 'AMS Days at CERN', 15-17 April, 2015.

57 O. Adriani, G. C. Barbarino, G. A. Bazilevskaya, et al. Measurement of Boron and Carbon Fluxes in Cosmic Rays with the PAMELA Experiment. Physics Reports, 544:323, 2014.

58 A. J. Davis, R. A. Mewaldt, W. R. Binns, et al. On the low energy decrease in galactic cosmic ray secondary/primary ratios. In R. A. Mewaldt, J. R. Jokipii, M. A. Lee, E. Möbius, and T. H. Zurbuchen, editors, Acceleration and Transport of Energetic Particles Observed in the Heliosphere, volume 528 of American Institute of Physics Conference Series, pages 421424, September 2000.

59 V. A. Derbina, V. I. Galkin, M. Hareyama, et al. Cosmic-Ray Spectra and Composition in the Energy Range of 10-1000 TeV per Particle Obtained by the RUNJOB Experiment. Astrophys. J. Lett. , 628:L41-L44, July 2005.

60 M. Amenomori and et al. International Cosmic Ray Conference, 2015.

61 T. Thambyahpillai. The Sidereal Diurnal Variation Measured Underground in London. International Cosmic Ray Conference, 3:383, August 1983.

62 D. B. Swinson and K. Nagashima. Corrected sidereal anisotropy for underground muons. Planet. Space Sci. , 33:1069-1072, September 1985.

63 K. Munakata, S. Yasue, S. Mori, et al. Two Hemisphere Observations of the North-South Sidereal Asymmetry at $1 \mathrm{TeV}$. International Cosmic Ray Conference, 4:639, 1995.

64 K. B. Fenton, A. G. Fenton, and J. E. Humble. Sidereal Variations at High Energies - Observations at Poatina. International Cosmic Ray Conference, 4:635, 1995.

65 M. Amenomori, S. Ayabe, X. J. Bi, et al. Anisotropy and Corotation of Galactic Cosmic Rays. Science, 314:439-443, October 2006.

66 R. Abbasi, Y. Abdou, T. Abu-Zayyad, et al. Observation of Anisotropy in the Galactic Cosmic-Ray Arrival Directions at $400 \mathrm{TeV}$ with IceCube. Astrophys. J. , 746:33, February 2012.

67 B. Bartoli, P. Bernardini, X. J. Bi, et al. ARGO-YBJ Observation of the Large-scale Cosmic Ray Anisotropy During the Solar Minimum between Cycles 23 and 24. Astrophys. J. , 809:90, August 2015.

68 T. K. Gaisser, T. Stanev, and S. Tilav. Cosmic ray energy spectrum from measurements of air showers. Frontiers of Physics, 8:748-758, December 2013.

69 CALET Collaboration. The CALET experiment on ISS. $\mathrm{Nu}$ clear Physics B Proceedings Supplements, 166:43-49, April 2007.

70 J. Chang et al. The DArk Matter Particle Explorer mission. Astropart. Phys., 2017.

71 Z. Cao. A future project at tibet: the large high altitude air shower observatory (LHAASO). Chinese Physics C, 34:249252, February 2010.

\section{Appendix}

We present the spectral parameters of all nuclei up to Iron as used in this work. 
Table 5. Low energy spectral parameters of all nuclei of the "modified poly-gonato" model (Sec. 2).

\begin{tabular}{ccccc}
\hline \hline$Z$ & $\begin{array}{c}E_{\mathrm{br}} / Z \\
\mathrm{GV}\end{array}$ & $\begin{array}{c}\Phi_{0} \\
\left(\mathrm{~m}^{-2} \mathrm{~s}^{-1} \mathrm{sr}^{-1} \mathrm{GeV}^{-1}\right)\end{array}$ & $\gamma_{1}$ & $\gamma_{2}$ \\
\hline 3 & 5.38 & $8.87 \times 10^{-3}$ & 0.25 & 2.73 \\
4 & 5.38 & $5.57 \times 10^{-3}$ & 0.25 & 2.94 \\
5 & 5.38 & $1.56 \times 10^{-2}$ & 0.25 & 3.44 \\
6 & 5.38 & $4.44 \times 10^{-2}$ & 0.25 & 2.85 \\
7 & 5.38 & $7.75 \times 10^{-3}$ & 0.25 & 2.91 \\
8 & 5.38 & $3.12 \times 10^{-2}$ & 0.25 & 2.87 \\
9 & 5.38 & $5.08 \times 10^{-4}$ & 0.25 & 2.88 \\
10 & 5.38 & $3.52 \times 10^{-3}$ & 0.25 & 2.83 \\
11 & 5.38 & $7.09 \times 10^{-4}$ & 0.25 & 2.85 \\
12 & 5.38 & $4.50 \times 10^{-3}$ & 0.25 & 2.83 \\
13 & 5.38 & $8.00 \times 10^{-4}$ & 0.25 & 2.85 \\
14 & 5.38 & $3.50 \times 10^{-3}$ & 0.25 & 2.94 \\
15 & 5.38 & $1.02 \times 10^{-4}$ & 0.25 & 2.88 \\
16 & 5.38 & $4.36 \times 10^{-4}$ & 0.25 & 2.74 \\
17 & 5.38 & $8.81 \times 10^{-5}$ & 0.25 & 2.87 \\
18 & 5.38 & $1.36 \times 10^{-4}$ & 0.25 & 2.83 \\
19 & 5.38 & $1.02 \times 10^{-4}$ & 0.25 & 2.84 \\
20 & 5.38 & $2.30 \times 10^{-4}$ & 0.25 & 2.89 \\
21 & 5.38 & $4.20 \times 10^{-5}$ & 0.25 & 2.83 \\
22 & 5.38 & $1.26 \times 10^{-4}$ & 0.25 & 2.80 \\
23 & 5.38 & $7.00 \times 10^{-5}$ & 0.25 & 2.82 \\
24 & 5.38 & $1.10 \times 10^{-4}$ & 0.25 & 2.86 \\
25 & 5.38 & $1.00 \times 10^{-4}$ & 0.25 & 2.71 \\
26 & 5.38 & $1.05 \times 10^{-3}$ & 0.25 & 2.73 \\
\hline \hline & & & &
\end{tabular}


Table 6. Injection spectral parameters $\gamma_{2}$ of all nuclei of the "spatially-dependent diffusion" model (Sec. 3).

\begin{tabular}{ccc}
\hline \hline Symbol & $Z$ & $\gamma_{2}$ \\
\hline $\mathrm{H}$ & 1 & 2.43 \\
$\mathrm{He}$ & 2 & 2.36 \\
$\mathrm{Li}$ & 3 & 2.26 \\
$\mathrm{Be}$ & 4 & 2.47 \\
$\mathrm{~B}$ & 5 & 2.67 \\
$\mathrm{C}$ & 6 & 2.38 \\
$\mathrm{~N}$ & 7 & 2.44 \\
$\mathrm{O}$ & 8 & 2.40 \\
$\mathrm{~F}$ & 9 & 2.41 \\
$\mathrm{Ne}$ & 10 & 2.36 \\
$\mathrm{Na}$ & 11 & 2.38 \\
$\mathrm{Mg}$ & 12 & 2.36 \\
$\mathrm{Al}$ & 13 & 2.38 \\
$\mathrm{Si}$ & 14 & 2.47 \\
$\mathrm{P}$ & 15 & 2.41 \\
$\mathrm{~S}$ & 16 & 2.27 \\
$\mathrm{Cl}$ & 17 & 2.40 \\
$\mathrm{Ar}$ & 18 & 2.36 \\
$\mathrm{~K}$ & 19 & 2.37 \\
$\mathrm{Ca}$ & 20 & 2.42 \\
$\mathrm{Sc}$ & 21 & 2.36 \\
$\mathrm{Ti}$ & 22 & 2.33 \\
$\mathrm{~V}$ & 23 & 2.35 \\
$\mathrm{Cr}$ & 24 & 2.39 \\
$\mathrm{Mu}$ & 25 & 2.18 \\
$\mathrm{Fe}$ & 26 & 2.31 \\
\hline \hline & &
\end{tabular}

